\title{
SPECIAL CLASSES OF THREE DIMENSIONAL AFFINE HYPERSPHERES CHARACTERIZED BY PROPERTIES OF THEIR CUBIC FORM.
}

\author{
LUC VRANCKEN
}

\begin{abstract}
It is well known that locally strongly convex affine hyperspheres can be determined as solutions of differential equations of Monge-Ampère type. The global properties of those solutions are well understood. However, due to the nature of the Monge-Ampère equation, not much is known about local solutions, particularly if the dimension of the hypersurface is greater then 2 . By the fundamental theorem, affine hyperspheres are completely determined by their metric $h$ and their difference tensor $K$ which together build the symmetric cubic form $C$. Following an idea of Bryant [1], we want to investigate affine hyperspheres for which at each point there exist isometries with respect to $h$ preserving this cubic form. The first non-trivial case is the case that $M$ is 3 -dimensional which is also the case which is investigated further in this paper.
\end{abstract}

Subject class: $53 \mathrm{~A} 15$

\section{INTRODUCTION}

In this paper we study nondegenerate affine hypersurfaces $M^{n}$ into $\mathbb{R}^{n+1}$, equipped with its standard affine connection $D$. It is well known that on such a hypersurface there exists a canonical transversal vector field $\xi$, which is called the affine normal. With respect to this transversal vector field one can decompose

$$
D_{X} Y=\nabla_{X} Y+h(X, Y) \xi
$$

thus introducing the affine metric $h$ and the induced affine connection $\nabla$. The Pick-Berwald theorem states that $\nabla$ coincides with the Levi Civita connection $\hat{\nabla}$ of the affine metric $h$ if and only if $M$ is immersed as a nondegenerate quadric. The difference tensor $K$ is introduced by

$$
K_{X} Y=\nabla_{X} Y-\hat{\nabla}_{X} Y
$$

It follows easily that $h(K(X, Y), Z)$ is symmetric in $X, Y$ and $Z$. The apolarity condition states that trace $K_{X}=0$ for every vector field $X$. The fundamental theorem of Dillen, Nomizu and the author, see [5] implies that an affine hypersurface is completely determined by the metric and the difference tensor $K$.

Deriving the affine normal, we introduce the affine shape operator $S$ by

$$
D_{X} \xi=-S X
$$

Here, we will restrict ourselves to the case that the affine shape operator $S$ is a multiple of the identity, i.e. $S=H I$. This means that all affine normals are parallel or pass through a fixed point. We will also assume that the metric is positive definite in which case one distinguishes the following classes of affine hyperspheres:

(i) elliptic affine hyperspheres, i.e. all affine normals pass through a fixed point and $H>0$,

(ii) hyperbolic affine hyperspheres, i.e. all affine normals pass through a fixed point and $H<0$,

(iii) parabolic affine hyperspheres, i.e. all the affine normals are parallel $(H=0)$.

Due to the work of amongst others Calabi [2], Pogorelov [15], Cheng and Yau [4], Sasaki [17] and Li [11], positive definite affine hyperspheres which are complete with respect to the affine metric $h$ are now well understood. In particular, the only complete elliptic or parabolic positive definite affine hyperspheres are respectively the ellipsoid and the paraboloid.

However, in the local case, one is far from obtaining a classification. The reason for this is that affine hyperspheres reduce to the study of the Monge-Ampère equations. In this paper we want to make a

Mathematisch Instituut, Universiteit Utrecht, Budapestlaan 6, 3584CD Utrecht, The Netherlands, Email: vrancken@math.uu.nl. 
distinction between different affine hyperspheres based on special properties of their difference tensor $K$. In order to do so, we apply an idea of Bryant [1] to the case of affine hyperspheres. Namely we want to make a distinction between different affine hyperspheres based on the number of orientation preserving isometries preserving the difference tensor $K$ at a given point.

In case that the dimension is two, it is easy to verify that either $K$ vanishes identically at a point or $K$ is preserved by rotations by an angle of $\frac{2 \pi}{3}$. So the first non trivial case is the case that the dimension of $M$ is 3 . This is the case that we will consider in this paper. At every point $p$ of $M$, we can introduce a symmetric polynomial $f_{p}$ by

$$
f_{p}(x, y, z)=h\left(K\left(x e_{1}+y e_{2}+z e_{3}, x e_{1}+y e_{2}+z e_{3}\right), x e_{1}+y e_{2}+z e_{3}\right),
$$

where $\left\{e_{1}, e_{2}, e_{3}\right\}$ is an orthonormal basis at the point $p$. The apolarity condition implies that the trace of this polynomial with respect to the metric vanishes. As far as such symmetric polynomials with vanishing trace on a 3 -dimensional real vector space are concerned, we quote the following result by Bryant:

Theorem 1. Let $p \in M$ and assume that there exist an orientation preserving isometry which preserves $f_{p}$. Then there exists an orthonormal basis of $T_{p} M$ such that either

(i) $f_{p}=0$, in this case $f_{p}$ is preserved by every isometry,

(ii) $f_{p}=\lambda\left(2 x^{3}-3 x y^{2}-3 x z^{2}\right)$, for some positive number $\lambda$ in which case $f_{p}$ is preserved by a 1-parameter group of rotations,

(iii) $f_{p}=6 \lambda x y z$ for some positive number $\lambda$, in which case $f_{p}$ is preserved by the discrete group $A_{4}$ of order 12,

(iv) $f_{p}=\lambda\left(x^{3}-3 x y^{2}\right)$ for some positive number $\lambda$, in which case $f_{p}$ is preserved by the discrete group $S_{3}$ of order 6

(v) $f_{p}=\lambda\left(2 x^{3}-3 x y^{2}-3 x z^{2}\right)+6 \mu x y z$, for some $\lambda, \mu>0$, with $\lambda \neq \mu$, in which case $f_{p}$ is preserved by the group $\mathbb{Z}_{2}$ of order 2 ,

(vi) $f_{p}=\lambda\left(2 x^{3}-3 x y^{2}-3 x z^{2}\right)+\mu\left(y^{3}-3 x y^{2}\right)$ for some $\lambda, \mu>0$, with $\mu \neq \sqrt{2} \lambda$, in which case $f_{p}$ is preserved by the group $\mathbb{Z}_{3}$.

In this paper, we will assume that one of the special cases of the above theorem is satisfied at every point of the hypersphere. The paper is organized as follows. In Section 2, we will deal with the case that at each point $p$, either Theorem 1(i) or (iii) is satisfied. The case that $M$ is an affine hypersphere satisfying either Theorem 1(ii) or Theorem 1(vi) is studied in Section 3, whereas the case that $M$ satisfies Theorem 1(iv), which also corresponds to Chen's equality studied in [18], [7] and [9], is considered in Section 4. Finally, the case that $M$ satisfies Theorem 1(v) at every point is investigated in Section 5 . We will call $M$ an affine hyperpshere of Type $\mathrm{k}$ if and only if Theorem $1(\mathrm{k})$ is satisfied at each point $p$ of $M$. To conclude this introduction, we remark that the basic integrability conditions for an affine hypersphere state that

$$
\begin{aligned}
& \widehat{R}(X, Y) Z=H(h(Y, Z) X-h(X, Z) Y)-\left[K_{X}, K_{Y}\right] Z \\
& \left(\hat{\nabla}_{X} K\right)(Y, Z)=\left(\hat{\nabla}_{Y} K\right)(X, Z) .
\end{aligned}
$$

Remark that by applying an affine transformation, we may always assume that $H=\epsilon$, where $\epsilon \epsilon$ $\{-1,0,1\}$.

\section{Affine hyperspheres of Type (i) or (iit)}

First, we remark that if $M$ is an affine hypersphere of Type (i) or Type (iii), there exists a local orthonormal basis $\left\{e_{1}, e_{2}, e_{3}\right\}$ such that

$$
\begin{array}{lll}
K\left(e_{1}, e_{2}\right)=\lambda e_{3}, & K\left(e_{2}, e_{3}\right)=\lambda e_{1}, & K\left(e_{3}, e_{1}\right)=\lambda e_{2}, \\
K\left(e_{1}, e_{1}\right)=0, & K\left(e_{2}, e_{2}\right)=0, & K\left(e_{3}, e_{3}\right)=0 .
\end{array}
$$

Substituting this in (4) implies that

$$
\widehat{R}(X, Y) Z=\left(H+\lambda^{2}\right)(h(Y, Z) X-h(X, Z) Y) .
$$

Consequently, Schur's lemma implies that $\lambda$ is a constant and that $M$ has constant sectional curvature. Thus, from the classification of positive definite affine spheres with constant sectional curvature, see [12], 
[8] or [19], it follows that $M$ is affine congruent with a positive definite quadric, if $\lambda=0$, or with the affine hypersphere described by $x_{1} \ldots x_{n+1}=1$, if $\lambda \neq 0$. Summarizing the above we have that

Theorem 2. Let $M$ be an affine hypersphere of Type (i). Then $M$ is affine congruent with a positive definite quadric.

Theorem 3. Let $M$ be an affine hypersphere of Type (iii). Then $M$ is affine congruent with the flat affine hypersphere described by $x_{1} \ldots x_{n+1}=1$.

\section{Affine hyperspheres of Type (ii) or (Vi)}

In this section, we assume that $M$ is an affine hypersphere of either Type (ii) or Type (vi). This means that at each point $p$ there exists an orthonormal basis $\left\{e_{1}, e_{2}, e_{3}\right\}$ such that

$$
\begin{array}{lll}
K\left(e_{1}, e_{1}\right)=2 \lambda e_{1}, & K\left(e_{1}, e_{2}\right)=-\lambda e_{1}, & K\left(e_{3}, e_{1}\right)=-\lambda e_{3}, \\
K\left(e_{2}, e_{2}\right)=-\lambda e_{1}+\mu e_{2}, & K\left(e_{2}, e_{3}\right)=-\mu e_{3}, & K\left(e_{3}, e_{3}\right)=-\lambda e_{1}-\mu e_{2},
\end{array}
$$

where $\lambda$ is a positive number and $\mu \neq \sqrt{2} \lambda$. If $M$ is an affine hypersphere of Type (ii) then $\mu=0$, whereas if $M$ is an affine hypersphere of Type (vi) then $\mu>0$.

Let $\left\{e_{1}, e_{2}, e_{3}\right\}$ denote the orthonormal basis constructed before, and denote by

$$
\widehat{\operatorname{Ric}}(Y, Z)=\frac{1}{2} \operatorname{trace}\{X \mapsto \widehat{R}(X, Y) Z\},
$$

the Ricci tensor associated with the affine metric $h$. Then, by a straightforward computation using (4) and the explicit expression for $K$ it follows that:

Lemma 1. Let $\left\{e_{1}, e_{2}, e_{3}\right\}$ be the orthonormal basis defined previously. Then it follows that

$$
\begin{array}{lll}
\widehat{\operatorname{Ric}}\left(e_{1}, e_{1}\right)=\epsilon+3 \lambda^{2}, & \widehat{\operatorname{Ric}}\left(e_{1}, e_{2}\right)=0, & \widehat{\operatorname{Ric}}\left(e_{3}, e_{1}\right)=0, \\
\widehat{\operatorname{Ric}}\left(e_{2}, e_{2}\right)=\epsilon+\lambda^{2}+\mu^{2}, & \widehat{\operatorname{Ric}}\left(e_{2}, e_{3}\right)=0, & \widehat{\operatorname{Ric}}\left(e_{3}, e_{3}\right)=\epsilon+\lambda^{2}+\mu^{2} .
\end{array}
$$

As $\mu^{2} \neq 2 \lambda^{2}$, we see as a consequence that the 1-1 symmetric tensor field $P$ associated with the Ricci tensor has at each point two different eigenvalues, one with multiplicity 1 , the other with multiplicity 2 . We then have

Lemma 2. Let $M$ be an affine hypersphere of Type (ii) or (vi) and let $p \in M$. Then there exist orthonormal vector fields $\left\{E_{1}, E_{2}, E_{3}\right\}$ defined on a neighborhood of the point $p$ and differentiable functions $\lambda$ and $\mu$, with $\mu^{2} \neq 2 \lambda^{2}$ and $\lambda>0$ such that

$$
\begin{array}{lll}
K\left(E_{1}, E_{1}\right)=2 \lambda E_{1}, & K\left(E_{1}, E_{2}\right)=-\lambda E_{1}, & K\left(E_{3}, E_{1}\right)=-\lambda E_{3}, \\
K\left(E_{2}, E_{2}\right)=-\lambda E_{1}+\mu E_{2}, & K\left(E_{2}, E_{3}\right)=-\mu E_{3}, & K\left(E_{3}, E_{3}\right)=-\lambda E_{1}-\mu E_{2} .
\end{array}
$$

Moreover, if $M$ is an affine hypersphere of Type (ii), then $\mu=0$, whereas if $M$ is an affine hypersphere of Type (vi), then $\mu>0$.

Proof. From Lemma 1 it follows that the eigenvalues of the differentiable operator $P$ have constant multiplicities. A standard result then implies that the eigendistributions are differentiable. We now take $E_{1}$ a local vector field spanning the 1-dimensional distribution and for $E_{2}$ and $E_{3}$ local orthonormal vector fields spanning the second distribution. As $E_{1}$ is uniquely determined, it follows that there exists a positive function $\lambda$ and differentiable functions $\mu$ and $\nu$ such that

$$
\begin{array}{lll}
K\left(E_{1}, E_{1}\right)=2 \lambda E_{1}, & K\left(E_{1}, E_{2}\right)=-\lambda E_{1}, & K\left(E_{3}, E_{1}\right)=-\lambda E_{3}, \\
K\left(E_{2}, E_{2}\right)=-\lambda E_{1}+\mu E_{2}-\nu E_{3}, & K\left(E_{2}, E_{3}\right)=-\nu E_{2}-\mu E_{3}, & K\left(E_{3}, E_{3}\right)=-\lambda E_{1}-\mu E_{2}+\nu E_{3} .
\end{array}
$$

If $M$ is an affine hypersphere of Type (ii) then $\mu^{2}+\nu^{2}=0$ and thus the proof is completed. Hence, we may assume that $M$ is an affine hypersphere of Type (vi) implying that $\mu^{2}+\nu^{2} \neq 0$. Then, if we define

$$
\begin{aligned}
& F_{2}=\cos \theta E_{2}+\sin \theta E_{3}, \\
& F_{3}=-\sin \theta E_{2}+\cos \theta E_{3},
\end{aligned}
$$


we see that the basis $\left\{E_{1}, F_{2}, F_{3}\right\}$ satisfies the conditions of the lemma provided that

$$
\begin{aligned}
& \cos 3 \theta \mu-\sin 3 \theta \nu>0 \\
& \cos 3 \theta \nu+\sin 3 \theta \mu=0 .
\end{aligned}
$$

As $\mu^{2}+\nu^{2} \neq 0$, it is clear that a differentiable function $\theta$ satisfying the above conditions exists.

In the remainder of this section, we will always work with the orthonormal basis constructed in the previous lemma. We then introduce local functions $a_{1}, \ldots, c_{3}$ by

$$
\begin{aligned}
& \hat{\nabla}_{E_{1}} E_{1}=a_{1} E_{2}+a_{2} E_{3}, \quad \hat{\nabla}_{E_{1}} E_{2}=-a_{1} E_{1}+a_{3} E_{3}, \quad \hat{\nabla}_{E_{1}} E_{3}=-a_{2} E_{1}-a_{3} E_{2}, \\
& \hat{\nabla}_{E_{2}} E_{1}=b_{1} E_{2}+b_{2} E_{3}, \quad \hat{\nabla}_{E_{2}} E_{2}=-b_{1} E_{1}+b_{3} E_{3}, \quad \hat{\nabla}_{E_{2}} E_{3}=-b_{2} E_{1}-b_{3} E_{2} \\
& \hat{\nabla}_{E_{3}} E_{1}=c_{1} E_{2}+c_{2} E_{3}, \quad \hat{\nabla}_{E_{3}} E_{2}=-c_{1} E_{1}+c_{3} E_{3}, \quad \hat{\nabla}_{E_{3}} E_{3}=-c_{2} E_{1}-c_{3} E_{2},
\end{aligned}
$$

We now will use the equations of Codazzi (5) and Gauss (4), in order to obtain more information about the above defined functions.

Lemma 3. Assume that $M$ is an affine hypersphere op Type (ii) or Type (vi). Denote by $\left\{E_{1}, E_{2}, E_{3}\right\}$ the corresponding orthonormal basis. Then, we have

$$
\begin{aligned}
& a_{2}=b_{2}=c_{1}=a_{1}=0, \\
& b_{2}=c_{1}, \\
& \mu a_{3}=0,
\end{aligned}
$$

and the functions $\lambda, \mu$ and $b_{1}$ satisfy the following system of differential equations:

$$
\begin{array}{lll}
E_{1}(\lambda)=-4 b_{1} \lambda, & E_{1}(\mu)=-\mu b_{1}, & E_{1}\left(b_{1}\right)=-\epsilon-b_{1}^{2}-3 \lambda^{2}, \\
E_{2}(\lambda)=0, & E_{2}(\mu)=-3 \mu c_{3}, & E_{2}\left(b_{1}\right)=0 \\
E_{3}(\lambda)=0, & E_{3}(\mu)=3 \mu b_{3}, & E_{3}\left(b_{1}\right)=0 .
\end{array}
$$

Proof. We compute $\left(\hat{\nabla}_{E_{1}} K\right)\left(E_{1}, E_{2}\right)=\left(\hat{\nabla}_{E_{2}} K\right)\left(E_{1}, E_{1}\right)$. On the one hand, we get that

$$
\begin{aligned}
\left(\hat{\nabla}_{E_{1}} K\right)\left(E_{1}, E_{2}\right) & =\hat{\nabla}_{E_{1}}\left(-\lambda E_{2}\right)-K\left(\hat{\nabla}_{E_{1}} E_{1}, E_{2}\right)-K\left(E_{1}, \hat{\nabla}_{E_{1}} E_{2}\right) \\
& =-E_{1}(\lambda) E_{2}+4 a_{1} \lambda E_{1}-a_{1} \mu E_{2}+a_{2} \mu E_{3},
\end{aligned}
$$

and on the other hand, we get that

$$
\begin{aligned}
\left(\hat{\nabla}_{E_{2}} K\right)\left(E_{1}, E_{1}\right) & =\hat{\nabla}_{E_{2}}\left(2 \lambda E_{1}\right)-2 K\left(\hat{\nabla}_{E_{2}} E_{1}, E_{1}\right) \\
& =2 E_{2}(\lambda) E_{1}+4 b_{1} \lambda E_{2}+4 b_{2} \lambda E_{3} .
\end{aligned}
$$

Comparing both sides it follows that

$$
\begin{aligned}
E_{1}(\lambda) & =-a_{1} \mu-4 b_{1} \lambda, \\
E_{2}(\lambda) & =2 a_{1} \lambda, \\
a_{2} \mu & =4 b_{2} \lambda .
\end{aligned}
$$


Similarly, using the other Codazzi equations, it follows that

$$
\begin{aligned}
\lambda\left(c_{1}-b_{2}\right) & =0, \\
\mu\left(b_{2}+c_{1}\right) & =-2 \lambda a_{2}=-2 \mu\left(3 a_{3}-b_{2}\right), \\
\mu\left(b_{1}-c_{2}\right) & =2 a_{1} \lambda, \\
4 \lambda\left(c_{2}-b_{1}\right) & =2 \mu a_{1}, \\
E_{3}(\lambda) & =2 \lambda a_{2}, \\
E_{2}(\lambda) & =-\mu\left(c_{2}-b_{1}\right), \\
E_{3}(\lambda) & =-\mu\left(b_{2}+c_{1}\right), \\
E_{1}(\mu) & =-\lambda a_{1}-\mu c_{2}, \\
E_{2}(\mu) & =-\lambda\left(b_{1}-c_{2}\right)-3 \mu c_{3}, \\
E_{3}(\mu) & =-\lambda\left(c_{1}-3 b_{2}\right)+3 \mu b_{3} .
\end{aligned}
$$

Moreover, computing all the components of the Gauss equation in a similar way, we also deduce that

$$
\begin{aligned}
& E_{2}\left(a_{1}\right)-E_{1}\left(b_{1}\right)=\epsilon+3 \lambda^{2}+a_{1}^{2}+b_{1}^{2}+b_{2} c_{1}-b_{2} a_{3}+a_{2} b_{3}-a_{3} c_{1}, \\
& E_{3}\left(a_{2}\right)-E_{1}\left(c_{2}\right)=\epsilon+3 \lambda^{2}+a_{2}^{2}+c_{2}^{2}+b_{2} c_{1}+b_{2} a_{3}-a_{1} c_{3}+a_{3} c_{1}, \\
& E_{2}\left(a_{2}\right)-E_{1}\left(b_{2}\right)=b_{1} a_{3}+b_{1} b_{2}+a_{1} a_{2}-a_{1} b_{3}+c_{2} b_{2}-c_{2} a_{3}, \\
& E_{3}\left(a_{1}\right)-E_{1}\left(c_{1}\right)=a_{2} c_{3}-a_{3} c_{2}+a_{1} a_{2}+b_{1} c_{1}+c_{1} c_{2}+b_{1} a_{3}, \\
& E_{2}\left(c_{2}\right)-E_{3}\left(b_{2}\right)=b_{1} c_{3}-b_{3} c_{1}-a_{2} b_{2}+a_{2} c_{1}-b_{2} b_{3}-c_{2} c_{3}, \\
& E_{2}\left(c_{1}\right)-E_{3}\left(b_{1}\right)=b_{3} c_{2}-c_{3} b_{2}+a_{1} c_{1}-a_{1} b_{2}-c_{1} c_{3}-b_{1} b_{3}, \\
& E_{3}\left(a_{3}\right)-E_{1}\left(c_{3}\right)=a_{1} c_{2}-a_{2} c_{1}+a_{2} a_{3}+a_{3} b_{3}+b_{3} c_{1}+c_{2} c_{3}, \\
& E_{1}\left(b_{3}\right)-E_{2}\left(a_{3}\right)=b_{1} a_{2}-a_{1} b_{2}-a_{1} a_{3}-b_{1} b_{3}+a_{3} c_{3}-c_{3} b_{2}, \\
& E_{3}\left(b_{3}\right)-E_{2}\left(c_{3}\right)=\epsilon-\lambda^{2}+2 \mu^{2}+b_{3}^{2}+c_{3}^{2}+a_{3} b_{2}-a_{3} c_{1}+b_{1} c_{2}-b_{2} c_{1} .
\end{aligned}
$$

As $\lambda \neq 0$, it follows first from (9) that $c_{1}=b_{2}$. We now consider 2 cases. First, we assume that $\mu=0$, then it follows from (11) and (10) that $a_{1}=a_{2}=0$. Combining (18) and (9), we get that $c_{1}=b_{2}=0$. The fact that $b_{1}=c_{2}$ now follows from (12). In the case that $\mu \neq 0$, we proceed as follows. As $c_{1}=b_{2}$ and $\mu \neq 0$, we deduce from (10) that $a_{3}=0$. As $\lambda \mu \neq 0$, it follows from combining (11) and (12) that $b_{1}=c_{2}$ and $a_{1}=0$. Similarly, it follows from combining (10) and (8) that $a_{2}=b_{2}=0$. The differential equations for $\lambda$ and $\mu$ now follow immediately from the remaining Codazzi equations. In order to obtain the differential equations for $b_{1}=c_{2}$, we use the Gauss equations (19), (23) and (24). These reduce to

$$
\begin{aligned}
& E_{1}\left(b_{1}\right)=-\epsilon-3 \lambda^{2}-b_{1}^{2}, \\
& E_{2}\left(b_{1}\right)=0, \\
& E_{3}\left(b_{1}\right)=0 .
\end{aligned}
$$

This completes the proof.

Remark that, as $\lambda>0$, the vector field $E_{1}$ is globally defined on $M$. As a consequence of the previous lemma, we see that $b_{1}$ is independent of the choice of $E_{2}$ and $E_{3}$ and is therefore globally defined on $M$. It also follows immediately from the previous lemma that the distributions $T_{1}=\operatorname{span}\left\{E_{2}, E_{3}\right\}$ and $T_{2}=\operatorname{span}\left\{E_{1}\right\}$ are integrable and orthogonal with respect to the affine metric $h$. We also get that $T_{2}$ is autoparallel and $T_{1}$ is spherical with mean curvature normal $-b_{1} E_{1}$. Therefore according to [10] we have that $(M, h)$ admits a warped product structure $M=\mathbb{R} \times_{e^{f}} N^{2}$ with $f: \mathbb{R} \rightarrow \mathbb{R}$ satisfying

$$
\begin{aligned}
& \frac{\partial f}{\partial t}=b_{1}, \\
& \frac{\partial b_{1}}{\partial t}=-\epsilon-b_{1}^{2}-3 \lambda^{2}, \\
& \frac{\partial \lambda}{\partial t}=-4 b_{1} \lambda,
\end{aligned}
$$


where $f, b_{1}$ and $\lambda$ only depend on the variable $t$, with $\frac{\partial}{\partial t}=E_{1}$ and the curvature of $N^{2}$ is given by

$$
K\left(N^{2}\right)=e^{2 f}\left(\epsilon-\lambda^{2}+2 \mu^{2}+b_{1}^{2}\right) .
$$

which we verify by a straightforward computation is indeed independent of $t$. Remark that it follows straightforward from the above differential equations that

$$
\frac{\partial}{\partial t}\left(\epsilon-\lambda^{2}+b_{1}^{2}\right)=-2 b_{1}\left(\epsilon-\lambda^{2}+b_{1}^{2}\right)
$$

implying that either $\epsilon-\lambda^{2}+b_{1}^{2}$ vanishes identically on $M$ or it vanishes nowhere on $M$. In the latter case, we may, by translating $f$, i.e. by replacing $N^{2}$ with a homothetic copy of itself, assume that $e^{2 f}\left(\epsilon-\lambda^{2}+b_{1}^{2}\right)=\tilde{\epsilon}$, where $\tilde{\epsilon}= \pm 1$. It is also clear that $U_{1}=e^{f} E_{2}$ and $U_{2}=e^{f} E_{3}$ form an orthonormal basis on $N^{2}$.

Next, we introduce a positive function $\beta$, depending only on the variable $t$, by the differential equation:

$$
\frac{\partial}{\partial t} \beta=\beta\left(b_{1}+\lambda\right)
$$

It then follows that

$$
\begin{aligned}
& D_{E_{1}}(\epsilon F+\xi)=0 \text {, } \\
& D_{E_{2}}(\epsilon F+\xi)=0 \text {, } \\
& D_{E_{3}}(\epsilon F+\xi)=0 \text {, } \\
& \left.D_{E_{1}}\left(\beta\left(-\left(b_{1}+\lambda\right) E_{1}+\xi\right)\right)=\beta\left(b_{1}+\lambda\right)\left(-\left(b_{1}+\lambda\right) E_{1}+\xi\right)\right)+\beta\left(\epsilon+b_{1}^{2}+3 \lambda^{2}\right) E_{1} \\
& -2 \beta\left(b_{1}+\lambda\right) \lambda E_{1}-\beta\left(b_{1}+\lambda\right) \xi+4 b_{1} \lambda \beta E_{1}-\epsilon \beta E_{1}=0, \\
& D_{E_{2}}\left(\beta\left(-\left(b_{1}+\lambda\right) E_{1}+\xi\right)\right)=-\beta\left(\epsilon+b_{1}^{2}-\lambda^{2}\right) E_{2}, \\
& D_{E_{3}}\left(\beta\left(-\left(b_{1}+\lambda\right) E_{1}+\xi\right)\right)=-\beta\left(\epsilon+b_{1}^{2}-\lambda^{2}\right) E_{3} \text {. }
\end{aligned}
$$

Now we consider different cases. First, we assume that $\epsilon+b_{1}^{2}-\lambda^{2}$ does not vanish identically on $M$, in which case we have seen that the function vanishes nowhere on $M$. We then have the following theorem:

Theorem 4. Let $\psi: N^{2} \rightarrow \mathbb{R}^{3}$ be a proper, positive definite affine hypersphere and let $\gamma: I \rightarrow \mathbb{R}^{2}$ be a curve. Let $\tilde{\epsilon}= \pm 1$ denote the mean curvature of the 2-dimensional affine hypersphere and define $F: I \times N^{2} \rightarrow \mathbb{R}^{3}:(t, u, v) \mapsto\left(\gamma_{1}(t) \psi(u, v), \gamma_{2}(t)\right)$.

(i) If $\gamma=\left(\gamma_{1}, \gamma_{2}\right)$ satisfies

- $\left(\gamma_{1}^{\prime} \gamma_{2}-\gamma_{2}^{\prime} \gamma_{1}\right) \gamma_{2} \neq 0$

- $\tilde{\epsilon} \gamma_{2} \gamma_{1}^{\prime}\left(\gamma_{1}^{\prime \prime} \gamma_{2}^{\prime}-\gamma_{2}^{\prime \prime} \gamma_{1}^{\prime}\right)<0$

- $\left(\gamma_{1} \gamma_{2}^{\prime}-\gamma_{1}^{\prime} \gamma_{2}\right)^{5}= - \pm 1 \gamma_{1}^{2}{\gamma_{2}^{\prime}}^{2}\left(\gamma_{1}^{\prime \prime} \gamma_{2}^{\prime}-\gamma_{2}^{\prime \prime} \gamma_{1}^{\prime}\right)$,

then $M$ is a 3-dimensional positive definite proper affine sphere,

(ii) If $\gamma=\left(\gamma_{1}, \gamma_{2}\right)$ satisfies

$$
\begin{aligned}
& \text { - } \gamma_{2} \gamma_{2}^{\prime} \neq 0 \\
& \text { - } \tilde{\epsilon} \gamma_{2} \gamma_{1}^{\prime}\left(\gamma_{1}^{\prime \prime} \gamma_{2}^{\prime}-\gamma_{2}^{\prime \prime} \gamma_{1}^{\prime}\right)>0 \\
& \text { - } \gamma_{1}^{\prime 5} \gamma_{1}^{4}= - \pm 1 \gamma_{2}^{\prime 2}\left(\gamma_{1}^{\prime \prime} \gamma_{2}^{\prime}-\gamma_{2}^{\prime \prime} \gamma_{1}^{\prime}\right)
\end{aligned}
$$

then $M$ is a 3 dimensional positive definite improper affine sphere.

Conversely every affine hypersphere of Type (ii) or (vi) satisfying $\epsilon+b_{1}^{2}+3 \lambda^{2} \neq 0$ can be obtained in this way.

Proof. As $\epsilon+b_{1}^{2}-\lambda^{2}$ vanishes nowhere on $M$, it follows from (32) to (34) that

$$
\phi=\beta\left(-\left(b_{1}+\lambda\right) E_{1}+\xi\right),
$$

defines a map from $N^{2}$ into $\mathbb{R}^{4}$. Moreover, it follows that

$$
\begin{aligned}
D_{E_{2}} \phi_{\star}\left(E_{2}\right) & =-\beta\left(\epsilon+b_{1}^{2}-\lambda^{2}\right) D_{E_{2}} E_{2} \\
& =-\beta\left(\epsilon+b_{1}^{2}-\lambda^{2}\right)\left(-\left(b_{1}+\lambda\right) E_{1}+\mu E_{2}+b_{3} E_{3}+\xi\right) \\
& =\mu \phi_{\star}\left(E_{2}\right)+b_{3} \phi_{\star}\left(E_{3}\right)-\left(\epsilon+b_{1}^{2}-\lambda^{2}\right) \phi \\
& =\mu \phi_{\star}\left(E_{2}\right)+b_{3} \phi_{\star}\left(E_{3}\right)-\tilde{\epsilon} e^{2 f} \phi .
\end{aligned}
$$


Similarly, we obtain that

$$
\begin{aligned}
& D_{E_{2}} \phi_{\star}\left(E_{3}\right)=-b_{3} \phi_{\star}\left(E_{2}\right)-\mu \phi_{\star}\left(E_{3}\right), \\
& D_{E_{3}} \phi_{\star}\left(E_{2}\right)=\left(c_{3}-\mu\right) \phi_{\star}\left(E_{3}\right), \\
& D_{E_{3}} \phi_{\star}\left(E_{3}\right)=\left(-c_{3}-\mu\right) \phi_{\star}\left(E_{2}\right)-\tilde{\epsilon} e^{2 f} \phi .
\end{aligned}
$$

The above implies that $\phi$ defines an immersion of $N^{2}$ as an equiaffine sphere in a linear subspace $\mathbb{R}^{3}$ of $\mathbb{R}^{4}$. The affine metric introduced by this immersion corresponds with the metric on $N^{2}$.

Next, we remark that if we put

$$
\delta=\frac{1}{\epsilon+b_{1}^{2}-\lambda^{2}}(\epsilon F+\xi)-\frac{\left(b_{1}+\lambda\right)}{\epsilon+b_{1}^{2}-\lambda^{2}}\left(E_{1}-\left(b_{1}-\lambda\right) F\right),
$$

it follows that

$$
D_{E_{2}} \delta=D_{E_{3}} \delta=0 .
$$

Hence $\delta$ depends only on the variable $t$.

Now, we consider two cases. First, we assume that $\epsilon \neq 0$. In this case, we may by applying a translation assume that $\xi=-\epsilon F$. Solving then (35) and (36) for $F$, we find that

$$
F(t, u, v)=\delta(t)-\frac{1}{\beta(t)\left(\epsilon+b_{1}^{2}-\lambda^{2}\right)} \phi(u, v),
$$

where $u$ and $v$ denote local coordinates on the surface $N^{2}$. As

$$
\begin{aligned}
D_{E_{1}}\left(E_{1}-\left(b_{1}-\lambda\right) F\right) & =2 \lambda E_{1}+\xi-\left(-\epsilon-b_{1}^{2}-3 \lambda^{2}+4 b_{1} \lambda\right) F-\left(b_{1}-\lambda\right) E_{1} \\
& =\left(3 \lambda-b_{1}\right) E_{1}+\left(b_{1}^{2}+3 \lambda^{2}-4 b_{1} \lambda\right) F \\
& =\left(3 \lambda-b_{1}\right)\left(E_{1}-\left(b_{1}-\lambda\right) F\right),
\end{aligned}
$$

it follows that $\delta^{\prime}$ and $\delta$ are proportional. As $F$ is linearly full this implies that $\delta$ and $\phi$ lie in mutually transversal subspaces. Consequently there exists a curve $\gamma=\left(\gamma_{1}, \gamma_{2}\right)$ in $\mathbb{R}^{2}$ such that after an affine transformation $F$ can be written as

$$
F(t, u, v)=\left(\gamma_{1}(t), \gamma_{2}(t) \phi(u, v)\right),
$$

where $\phi$ is a positive definite proper affine hypersphere. It follows that

$$
\begin{aligned}
& F_{t}=\left(\gamma_{1}^{\prime}, \gamma_{2}^{\prime} \phi\right) \\
& F_{u}=\left(0, \gamma_{2} \phi_{u}\right) \\
& F_{v}=\left(0, \gamma_{2} \phi_{v}\right) .
\end{aligned}
$$

As $F$ is an immersion and $F$ itself is a transversal vector field it follows that $\gamma_{2} \neq 0$ and $\left(\gamma_{1}^{\prime} \gamma_{2}-\gamma_{2}^{\prime} \gamma_{1}\right) \neq 0$. We also have that

$$
\begin{aligned}
F_{t t} & =\left(\gamma_{1}^{\prime \prime}, \gamma_{2}^{\prime \prime} \phi\right)=\ldots F_{t}+\frac{\left(\gamma_{1}^{\prime \prime} \gamma_{2}^{\prime}-\gamma_{2}^{\prime \prime} \gamma_{1}^{\prime}\right)}{\left(\gamma_{1} \gamma_{2}^{\prime}-\gamma_{1}^{\prime} \gamma_{2}\right)} F, \\
F_{t u} & =\left(0, \gamma_{2}^{\prime} \phi_{u}\right)=\frac{\gamma_{2}^{\prime}}{\gamma_{2}} F_{u}, \\
F_{t v} & =\left(0, \gamma_{2}^{\prime} \phi_{v}\right)=\frac{\gamma_{2}^{\prime}}{\gamma_{2}} F_{v}, \\
F_{u u} & =\left(0, \gamma_{2} \phi_{u u}\right)=\ldots F_{u}+\ldots F_{v}+\ldots F_{t}-\tilde{\epsilon} g\left(\phi_{u}, \phi_{u}\right) \frac{\gamma_{2} \gamma_{1}^{\prime}}{\left(\gamma_{1}^{\prime} \gamma_{2}-\gamma_{1} \gamma_{2}^{\prime}\right)} F, \\
F_{u v} & =\left(0, \gamma_{2} \phi_{u v}\right)=\ldots F_{u}+\ldots F_{v}+\ldots F_{t}-\tilde{\epsilon} g\left(\phi_{u}, \phi_{v}\right) \frac{\gamma_{2} \gamma_{1}^{\prime}}{\left(\gamma_{1}^{\prime} \gamma_{2}-\gamma_{1} \gamma_{2}^{\prime}\right)} F, \\
F_{v v} & =\left(0, \gamma_{2} \phi_{v v}\right)=\ldots F_{u}+\ldots F_{v}+\ldots F_{t}-\tilde{\epsilon} g\left(\phi_{v}, \phi_{v}\right) \frac{\gamma_{2} \gamma_{1}^{\prime}}{\left(\gamma_{1}^{\prime} \gamma_{2}-\gamma_{1} \gamma_{2}^{\prime}\right)} F,
\end{aligned}
$$

where $g$ denotes the affine metric on the 2-dimensional affine hypersphere. Hence taking into account that $\phi$ is an equiaffine sphere with mean curvature $\tilde{\epsilon}$, it follows that $F$ defines an equiaffine hypersphere if and only if

$$
\gamma_{2}^{4}\left(\gamma_{1} \gamma_{2}^{\prime}-\gamma_{1}^{\prime} \gamma_{2}\right)^{5}= \pm 1 \gamma_{2}^{2}{\gamma_{1}^{\prime}}^{2}\left(\gamma_{1}^{\prime \prime} \gamma_{2}^{\prime}-\gamma_{2}^{\prime \prime} \gamma_{1}^{\prime}\right),
$$

whereas the condition that the induced metric is positive definite implies that

$$
\left(\gamma_{1}^{\prime \prime} \gamma_{2}^{\prime}-\gamma_{2}^{\prime \prime} \gamma_{1}^{\prime}\right) \tilde{\epsilon} \gamma_{2} \gamma_{1}^{\prime}<0
$$


This completes the proof in this case. In the case that $\epsilon=0$, we proceed as follows. We first remark that (36) reduces to

$$
\begin{aligned}
\delta(t) & =\frac{1}{b_{1}^{2}-\lambda^{2}} \xi-\frac{1}{b_{1}-\lambda} E_{1}+F \\
& =\frac{1}{b_{1}^{2}-\lambda^{2}}\left(\xi-\left(b_{1}+\lambda\right) E_{1}\right)+F \\
& =\frac{1}{\beta\left(b_{1}^{2}-\lambda^{2}\right)} \phi+F .
\end{aligned}
$$

Hence, we still have that

$$
F(t, u, v)=\delta(t)-\frac{1}{\beta\left(b_{1}^{2}-\lambda^{2}\right)} \phi
$$

As

$$
\begin{aligned}
\delta^{\prime}(t) & =E_{1}-\frac{1}{\beta\left(b_{1}-\lambda\right)} \phi+\frac{2 b_{1}}{\beta\left(b_{1}^{2}-\lambda^{2}\right)} \phi \\
& =E_{1}+\frac{1}{\beta\left(b_{1}+\lambda\right)} \phi \\
& =E_{1}+\frac{1}{\beta\left(b_{1}+\lambda\right)}\left(\beta \xi-\beta\left(b_{1}+\lambda\right) E_{1}\right) \\
& =\frac{1}{b_{1}+\lambda} \xi
\end{aligned}
$$

and by an affine transformation we may assume that $\xi=(1,0,0,0)$, it still follows that if necessary after applying a translation we may assume that there exists a curve $\gamma=\left(\gamma_{1}, \gamma_{2}\right)$ in $\mathbb{R}^{2}$ such that

$$
F(t, u, v)=\left(\gamma_{1}(t), \gamma_{2}(t) \phi(u, v)\right)
$$

where $\phi: N^{2} \rightarrow \mathbb{R}^{3}$ is a positive definite affine hypersphere. It follows that

$$
\begin{aligned}
& F_{t}=\left(\gamma_{1}^{\prime}, \gamma_{2}^{\prime} \phi\right), \\
& F_{u}=\left(0, \gamma_{2} \phi_{u}\right), \\
& F_{v}=\left(0, \gamma_{2} \phi_{v}\right) .
\end{aligned}
$$

As $(1,0,0,0)$ is a transversal vector field it follows that $\gamma_{2} \gamma_{2}^{\prime} \neq 0$. We also have that

$$
\begin{aligned}
& F_{t t}=\left(\gamma_{1}^{\prime \prime}, \gamma_{2}^{\prime \prime} \phi\right)=\ldots F_{t}+\frac{\left(\gamma_{1}^{\prime \prime} \gamma_{2}^{\prime}-\gamma_{2}^{\prime \prime} \gamma_{1}^{\prime}\right)}{\gamma_{2}^{\prime}} \xi, \\
& F_{t u}=\left(0, \gamma_{2}^{\prime} \phi_{u}\right)=\frac{\gamma_{2}^{\prime}}{\gamma_{2}} F_{u}, \\
& F_{t v}=\left(0, \gamma_{2}^{\prime} \phi_{v}\right)=\frac{\gamma_{2}^{\prime}}{\gamma_{2}} F_{v}, \\
& F_{u u}=\left(0, \gamma_{2} \phi_{u u}\right)=\ldots F_{u}+\ldots F_{v}+\ldots F_{t}+\tilde{\epsilon} g\left(\phi_{u}, \phi_{u}\right) \frac{\gamma_{2} \gamma_{1}^{\prime}}{\gamma_{2}^{\prime}} \xi, \\
& F_{u v}=\left(0, \gamma_{2} \phi_{u v}\right)=\ldots F_{u}+\ldots F_{v}+\ldots F_{t}+\tilde{\epsilon} g\left(\phi_{u}, \phi_{v}\right) \frac{\gamma_{2} \gamma_{1}^{\prime}}{\gamma_{2}^{\prime}} \xi, \\
& F_{v v}=\left(0, \gamma_{2} \phi_{v v}\right)=\ldots F_{u}+\ldots F_{v}+\ldots F_{t}+\tilde{\epsilon} g\left(\phi_{v}, \phi_{v}\right) \frac{\gamma_{2} \gamma_{1}^{\prime}}{\gamma_{2}^{\prime}} \xi .
\end{aligned}
$$

Hence taking into account that $\phi$ is an equiaffine sphere with mean curvature $\tilde{\epsilon}$, it follows that $F$ defines an improper equiaffine hypersphere with affine normal $(1,0,0,0)$ if and only if

$$
{\gamma_{2}^{\prime}}^{5} \gamma_{2}^{2}= \pm 1{\gamma_{1}^{\prime}}^{2}\left(\gamma_{1}^{\prime \prime} \gamma_{2}^{\prime}-\gamma_{2}^{\prime \prime} \gamma_{1}^{\prime}\right)
$$

whereas the condition that the induced metric is positive definite and non degenerate implies that

$$
\left(\gamma_{1}^{\prime \prime} \gamma_{2}^{\prime}-\gamma_{2}^{\prime \prime} \gamma_{1}^{\prime}\right) \tilde{\epsilon} \gamma_{2} \gamma_{1}^{\prime}>0
$$

which completes the proof of the theorem.

Next we consider that $\epsilon= \pm 1$ and $\epsilon+b_{1}^{2}-\lambda^{2}$ vanishes identically on $M$. We then have the following theorem:

Theorem 5. Let $\psi: N^{2} \rightarrow \mathbb{R}^{3}:(u, v) \mapsto(u, v, f(u, v))$ be an improper positive definite affine hypersphere with affine normal $(0,0,1)$ and let $\gamma: I \rightarrow \mathbb{R}$ be a curve satisfying

$$
\frac{\left(\gamma_{1} \gamma_{2}^{\prime}-\gamma_{2} \gamma_{1}^{\prime}\right)^{5} \gamma_{1}^{2}}{\left(\gamma_{1}^{\prime} \gamma_{2}^{\prime \prime}-\gamma_{2}^{\prime} \gamma_{1}^{\prime \prime}\right) \gamma_{1}^{\prime 2}}=c
$$


where $c$ is a non-zero constant. Then, $F: I \times N^{2} \rightarrow \mathbb{R}^{3}:(t, u, v) \mapsto\left(\gamma_{1} u, \gamma_{1} v, \gamma_{1} f(u, v)+\gamma_{2}, \gamma_{1}\right)$ defines a proper affine hypersphere, which is positive definite provided

$$
\begin{array}{r}
\left(\gamma_{1}^{\prime} \gamma_{2}-\gamma_{1} \gamma_{2}^{\prime}\right) \neq 0, \\
\left(\gamma_{1}^{\prime} \gamma_{2}^{\prime \prime}-\gamma_{1}^{\prime \prime} \gamma_{2}^{\prime}\right) \gamma_{1}^{\prime} \gamma_{1}>0 .
\end{array}
$$

Conversely every proper affine hypersphere of Type (ii) or (vi) satisfying $\epsilon+b_{1}^{2}-\lambda^{2}=0$ can be obtained in this way.

Proof. From (28) to (34) it follows that we can introduce constant vectors $C_{1}$ and $C_{2}$ such that

$$
\begin{aligned}
& \epsilon F+\xi=C_{1}, \\
& \beta\left(-\left(b_{1}+\lambda\right) E_{1}+\xi\right)=C_{2} .
\end{aligned}
$$

Hence it follows that

$$
\beta\left(-\left(b_{1}+\lambda\right) E_{1}+C_{1}-\epsilon F\right)=C_{2},
$$

or equivalently

$$
\left(b_{1}+\lambda\right) E_{1}=-C_{2} \beta^{-1}+C_{1}-\epsilon F .
$$

Using that $\epsilon+b_{1}^{2}-\lambda^{2}=0$, we can rewrite the above as

$$
\frac{\partial}{\partial t} F=\left(b_{1}-\lambda\right) F-\epsilon C_{1}\left(b_{1}-\lambda\right)+C_{2} \epsilon\left(b_{1}-\lambda\right) \beta^{-1} .
$$

We now fix an initial value $t_{0}$. As mentioned before, we know that the distribution $T_{1}$ is integrable. Let $N_{0}$ denote the integral manifold through $t_{0}$. As

$$
\begin{aligned}
& D_{E_{2}} E_{2}=-\left(b_{1}+\lambda\right) E_{1}+\mu E_{2}+b_{3} E_{3}+\xi \\
& D_{E_{2}} E_{3}=-b_{3} E_{2}-\mu E_{3}, \\
& D_{E_{3}} E_{2}=\left(c_{3}-\mu\right) E_{3}, \\
& D_{E_{3}} E_{3}=\left(-c_{3}-\mu\right) E_{2}-\left(b_{1}+\lambda\right) E_{1}+\xi .
\end{aligned}
$$

and

$$
D_{E_{2}}\left(-\left(b_{1}+\lambda\right) E_{1}+\xi\right)=0=D_{E_{3}}\left(-\left(b_{1}+\lambda\right) E_{1}+\xi\right)
$$

it follows that this integral manifold is contained as an improper affine sphere in a 3-dimensional affine subspace of $\mathbb{R}^{4}$ with affine normal a multiple of $C_{2}$. Moreover, choosing the initial conditions for $\beta$ appropriately, we may assume that the affine normal actually is $C_{2}$. Hence by applying a translation and an affine transformation, we get that

$$
F\left(t_{0}, u, v\right)=(u, v, f(u, v), 0),
$$

where $(u, v) \mapsto(u, v, f(u, v)$ defines an improper affine sphere with affine normal $(0,0,1)$.

As the immersion $F$ itself is nondegenerate it follows from (40) and (41) that the vector $C_{1}$ is transversal to the space spanned by $F\left(t_{0}, u, v\right)$. Therefore by applying an affine transformation, it follows that $C_{1}=(0,0,0,1)$. We then get immediately from (40) and (41) that there exists a curve $\gamma=\left(\gamma_{1}, \gamma_{2}\right)$ such that

$$
F(t, u, v)=\left(\gamma_{1} u, \gamma_{1} v, \gamma_{1} f(u, v)+\gamma_{2}, \gamma_{1}\right)
$$

It then follows that

$$
\begin{aligned}
& F_{t}=\left(\gamma_{1}^{\prime} u, \gamma_{1}^{\prime} v, \gamma_{1}^{\prime} f(u, v)+\gamma_{2}^{\prime}, \gamma_{1}^{\prime}\right), \\
& F_{u}=\left(\gamma_{1}, 0, \gamma_{1} f_{u}, 0\right) \\
& F_{v}=\left(0, \gamma_{1}, \gamma_{1} f_{v}, 0\right)
\end{aligned}
$$


and

$$
\begin{aligned}
F_{u u} & =\gamma_{1} f_{u u}(0,0,1,0), \\
F_{u v} & =\gamma_{1} f_{u v}(0,0,1,0), \\
F_{v v} & =\gamma_{1} f_{v v}(0,0,1,0), \\
F_{u t} & =\frac{\gamma_{1}^{\prime}}{\gamma_{1}} F_{u}, \\
F_{v t} & =\frac{\gamma_{1}^{\prime}}{\gamma_{1}} F_{v}, \\
F_{t t} & =\ldots F_{t}+\frac{\left(\gamma_{1}^{\prime} \gamma_{2}^{\prime \prime}-\gamma_{1}^{\prime \prime} \gamma_{2}^{\prime}\right)}{\gamma_{1}^{\prime}}(0,0,1,0) .
\end{aligned}
$$

As

$$
(0,0,1,0)=\frac{\gamma_{1}^{\prime} F-\gamma_{1} F_{t}}{\left(\gamma_{1}^{1} \gamma_{2}-\gamma_{1} \gamma_{2}^{\prime}\right)},
$$

we see that the hypersurfaces defined by (42) defines an affine sphere provided that there exist a constant $c$ such that

$$
\frac{\gamma_{1}^{2}\left(\gamma_{2} \gamma_{1}^{\prime}-\gamma_{2}^{\prime} \gamma_{1}\right)^{5}}{\gamma_{1}^{\prime 2}\left(\gamma_{1}^{\prime \prime} \gamma_{2}^{\prime}-\gamma_{1}^{\prime} \gamma_{2}^{\prime \prime}\right)}=c
$$

where, in order for the immersion to be positive definite and nondegenerate, $\gamma$ satisfies moreover:

$$
\begin{aligned}
&\left(\gamma_{1}^{\prime} \gamma_{2}-\gamma_{1} \gamma_{2}^{\prime}\right) \neq 0, \\
&\left(\gamma_{1}^{\prime} \gamma_{2}^{\prime \prime}-\gamma_{1}^{\prime \prime} \gamma_{2}^{\prime}\right) \gamma_{1}^{\prime} \gamma_{1}>0 .
\end{aligned}
$$

Finally we deal with the case that $M$ is an improper affine hypersphere such that $b_{1}^{2}-\lambda^{2}=0$. Those hyperspheres are obtained as in the next theorem:

Theorem 6. Let $\psi: N^{2} \rightarrow \mathbb{R}^{3}:(u, v) \mapsto(u, v, f(u, v))$ be a positive definite improper affine sphere with affine normal $(0,0,1)$. Then

$$
\begin{aligned}
& F(t, u, v)=\left(u t, v t, t, f(u, v) t+c t^{4}\right), \\
& F(t, u, v)=\left(u, v, f(u, v)+c t^{3}, t^{4}\right),
\end{aligned}
$$

where $t>0$ and $c$ is a positive constant, define improper affine hyperspheres of Type (ii) or (vi). Conversely every improper affine hypersphere of Type (ii) or (vi) satisfying $b_{1}^{2}-\lambda^{2}=0$ can be locally obtained in this way.

Proof. As $b_{1}^{2}-\lambda^{2}=0$ and $\lambda>0$, we have two cases to consider. First, we assume that $b_{1}=\lambda>0$. In this case, the differential equation for $\beta$ states that

$$
\frac{\partial}{\partial t} \beta=2 b_{1} \beta,
$$

from which we deduce that we can take $\beta=\frac{1}{\sqrt{b_{1}}}$. Proceeding as in the previous theorem, we find that there exist constant vectors $C_{1}$ and $C_{2}$, where $C_{2}$ is a multiple of $(0,0,1,0)$ and $C_{1}$ is a multiple of $(0,0,0,1)$ such that

$$
\frac{\partial}{\partial t} F=-\frac{1}{2 \sqrt{b_{1}}} C_{2}+\frac{1}{2 b_{1}} C_{1},
$$

and such that the integral surface through the point $t_{0}$ is given by

$$
F\left(t_{0}, u, v\right)=(u, v, f(u, v), 0),
$$

where $(u, v) \mapsto(u, v, f(u, v))$ describes an improper affine sphere with affine normal $(0,0,1)$. Combining (43) and (44) it is then clear that there exists a curve $\gamma=\left(\gamma_{1}, \gamma_{2}\right)$ such that

$$
F(t, u, v)=\left(u, v, f(u, v)+\gamma_{1}(t), \gamma_{2}(t)\right) .
$$

As

$$
\begin{aligned}
& F_{t}=\left(0,0, \gamma_{1}^{\prime}, \gamma_{2}^{\prime}\right), \\
& F_{u}=\left(1,0, f_{u}, 0\right), \\
& F_{v}=\left(0,1, f_{v}, 0\right),
\end{aligned}
$$


and

$$
\begin{aligned}
F_{u u} & =f_{u u}(0,0,1,0)=\ldots F_{t}-f_{u u} \frac{\gamma_{2}^{\prime}}{\gamma_{1}^{\prime}}(0,0,0,1), \\
F_{u v} & =f_{u v}(0,0,1,0)=\ldots F_{t}-f_{u v} \frac{\gamma_{2}^{\prime}}{\gamma_{1}^{\prime}}(0,0,0,1), \\
F_{v v} & =f_{v v}(0,0,1,0)=\ldots F_{t}-f_{v v} \frac{\gamma_{2}^{\prime}}{\gamma_{1}^{\prime}}(0,0,0,1), \\
F_{u t} & =0, \\
F_{v t} & =0, \\
F_{t t} & =\ldots F_{t}+\frac{\left(\gamma_{2}^{\prime \prime} \gamma_{1}^{\prime}-\gamma_{1}^{\prime \prime} \gamma_{2}^{\prime}\right)}{\gamma_{1}^{\prime}}(0,0,0,1),
\end{aligned}
$$

we see that $F$ defines an improper affine sphere with affine normal a constant multiple of $(0,0,0,1)$ if and only if

$$
\frac{\left(\gamma_{2}^{\prime \prime} \gamma_{1}^{\prime}-\gamma_{1}^{\prime \prime} \gamma_{2}^{\prime}\right) \gamma_{2}^{\prime 2}}{\gamma_{1}^{\prime 5}}=\tilde{c}
$$

where $\tilde{c}$ is a constant. It then follows by reparameterizing such that $\gamma_{1}(t)=t$, if necessary after applying a translation, that $\gamma_{2}(t)=c t^{\frac{4}{3}}$.

Next, we consider the case that $b_{1}=-\lambda$. By applying an affine transformation, we may assume that $\xi=(0,0,0,1)$. Proceeding as before, we obtain that

$$
F\left(t_{0}, u, v\right)=(u, v, 0, f(u, v)),
$$

where $(u, v) \mapsto(u, v, f(u, v))$ defines an improper affine sphere with affine normal $(0,0,1)$. As the function $\lambda$ is deterermined by

$$
\frac{\partial}{\partial t} \lambda=4 \lambda^{2}>0
$$

it follows that after a translation of the $t$-variable, we may assume that $\lambda(t)=-\frac{1}{4 t}$. As $D_{E_{1}} E_{1}=$ $2 \lambda E_{1}+\xi$,we get that $F$ is determined by the differential equation:

$$
\frac{\partial^{2}}{\partial t^{2}} F=-\frac{1}{2 t} \frac{\partial}{\partial t} F+\xi
$$

with initial condition $F\left(t_{0}, u, v\right)=(u, v, 0, f(u, v))$. As $E_{1}-\left(b_{1}-\lambda\right) F$ is constant along and transversal to the integral submanifold we may, if necessary after applying an affine transformation, take as second initial condition that

$$
E_{1}\left(t_{0}, u, v\right)+2 \lambda\left(t_{0}\right) F\left(t_{0}, u, v\right)=(0,0,1,0) .
$$

As it follows from (46) that the map $t \mapsto \lambda$ is a diffeomorphism, we can take $\lambda$ as a variable. Then the differential equations reduce to

$$
16 \lambda^{4} \frac{\partial^{2} F}{\partial \lambda^{2}}+24 \lambda^{3} \frac{\partial F}{\partial \lambda}=\xi,
$$

with initial conditions at the point $\lambda_{0}$ given by

$$
\begin{aligned}
& F\left(\lambda_{0}, u, v\right)=(u, v, 0, f(u, v)), \\
& \frac{\partial F}{\partial \lambda}\left(\lambda_{0}, u, v\right)=\left(-\frac{u}{2 \lambda_{0}},-\frac{v}{2 \lambda_{0}}, \frac{1}{4 \lambda_{0}^{2}},-\frac{f(u, v)}{2 \lambda_{0}}\right) .
\end{aligned}
$$

Solving the above differential equation (48), we find that

$$
F(\lambda, u, v)=C_{1}(u, v) \lambda^{-\frac{1}{2}}+C_{2}(u, v)+\frac{1}{48} \lambda^{-2} \xi .
$$

Comparing now with the initial conditions it follows that

$$
\begin{aligned}
& C_{2}(u, v)=\left(0,0, \frac{1}{2 \lambda_{0}}, \frac{3}{48} \lambda_{0}^{-2}\right) \\
& C_{1}(u, v)=\left(u \lambda_{0}^{\frac{1}{2}}, v \lambda_{0}^{\frac{1}{2}},-\frac{1}{2} \lambda_{0}^{-\frac{1}{2}}, f(u, v) \lambda_{0}^{\frac{1}{2}}-\frac{1}{12} \lambda_{0}^{-\frac{3}{2}}\right) .
\end{aligned}
$$

Hence applying an affine transformation and a change of variables it is then easy to check that $F$ is congruent with an open part of

$$
F(t, u, v)=\left(u t, v t, t, f(u, v) t+d t^{4}\right)
$$


Remark 1. It is actually quite surprising that a 3-dimensional affine hypersphere of any type (elliptic, hyperbolic or improper) can be constructed starting from a 2-dimensional affine hypersphere of arbitrary type. In particular, a 3-dimensional elliptic affine hypersphere can be constructed starting from a 2dimensional hyperbolic affine hypersphere. This is not the case when studying minimal Lagrangian submanifolds of the complex projective space, see [16]. There, it is shown that in order to obtain a minimal Lagrangian submanifold for which the second fundamental form has a similar form as the difference tensor in Type (ii) or (vi) one has to start from a minimal Lagrangian submanifold in $\mathbb{C} P^{2}(4)$.

Remark 2. If in the previous theorem, we assume that the starting hypersphere is hyperbolic, we see that a special curve $\gamma$ is given by

$$
\gamma(t)=\left(\frac{1}{2} e^{t}, \frac{1}{2} e^{-t}\right)
$$

In this case, the resulting solution is the well known Calabi product of hyperbolic affine hyperspheres, see also [6] and [13].

Remark 3. It is well known that a 2-dimensional positive definite improper affine hypersphere can be locally written as

$$
\left(\frac{1}{2}(z-\bar{G}), \frac{1}{8}(z \bar{z}-G \bar{G})+\frac{1}{2} \operatorname{Re}\left(\int G\right)-\frac{1}{4} \operatorname{Re}(G(z) z)\right)
$$

where $G$ is a holomorphic function. Combining this with the previous theorems yields many explicit examples of 3 -dimensional positive definite affine hyperspheres.

\section{Affine hyperspheres of Type (iv)}

In this section, we assume that $M$ is an affine hypersphere of Type (iv). This means that at each point $p$ there exists an orthonormal basis $\left\{e_{1}, e_{2}, e_{3}\right\}$ such that

$$
\begin{aligned}
& K\left(e_{1}, e_{1}\right)=\lambda e_{1}, \quad K\left(e_{1}, e_{2}\right)=-\lambda e_{2}, \quad K\left(e_{3}, e_{1}\right)=0, \\
& K\left(e_{2}, e_{2}\right)=-\lambda e_{1}, \quad K\left(e_{2}, e_{3}\right)=0, \quad K\left(e_{3}, e_{3}\right)=0,
\end{aligned}
$$

where $\lambda$ is a positive number. From the above expression, assuming that $\epsilon= \pm 1$, it is clear that $M$ realizes at every point the equality in the following inequality, which was derived in [18] and which states

$$
3 \hat{\kappa}(p)-\sup _{\Pi \in G_{2}\left(T_{x} M\right)} \hat{K}_{p}(\Pi) \geq 2 \epsilon .
$$

Here $\hat{\kappa}$ and $\hat{K}$ denote respectively the normalized scalar curvature and sectional curvature. Furthermore, $G_{2}\left(T_{x} M\right)$ denotes the Grassmannian of 2-dimensional subspaces of $T_{x} M$. This inequality was motivated by the work of Chen for submanifolds of real space forms ([3]). It was shown in [18] that a 3-dimensional proper affine hypersphere realizes at every point the equality if and only if $M$ is a proper affine hypersphere of Type (iv). As 3-dimensional elliptic and hyperbolic affine hyperspheres realizing the equality were respectively classified in [9] and [7], we will restrict ourselves in the remainder of this section to the case that $M$ is an improper affine sphere, i.e. $\epsilon=0$.

First, we remark that a straightforward computation shows the following

Lemma 4. Let $\left\{e_{1}, e_{2}, e_{3}\right\}$ be the orthonormal basis defined previously. Then it follows that

$$
\begin{array}{lll}
\widehat{\operatorname{Ric}}\left(e_{1}, e_{1}\right)=\lambda^{2}, & \widehat{\operatorname{Ric}}\left(e_{1}, e_{2}\right)=0, & \widehat{\operatorname{Ric}}\left(e_{3}, e_{1}\right)=0, \\
\widehat{\operatorname{Ric}}\left(e_{2}, e_{2}\right)=\lambda^{2}, & \widehat{\operatorname{Ric}}\left(e_{2}, e_{3}\right)=0, & \widehat{\operatorname{Ric}}\left(e_{3}, e_{3}\right)=0 .
\end{array}
$$

We again see as a consequence that the 1-1 symmetric tensor field $P$ associated with the Ricci tensor has at each point two different eigenvalues, one with multiplicity 1 , the other with multiplicity 2 . As in the previous section we then can show that there exist orthonormal vector fields $\left\{E_{1}, E_{2}, E_{3}\right\}$ defined on a neighborhood of the point $p$ and a non vanishing differentiable functions $\lambda$, such that

$$
\begin{array}{lll}
K\left(E_{1}, E_{1}\right)=\lambda E_{1}, & K\left(E_{1}, E_{2}\right)=-\lambda E_{2}, & K\left(E_{3}, E_{1}\right)=0, \\
K\left(E_{2}, E_{2}\right)=-\lambda E_{1}, & K\left(E_{2}, E_{3}\right)=0, & K\left(E_{3}, E_{3}\right)=0 .
\end{array}
$$

Remark that the choice of $E_{1}$ and $E_{2}$ is not unique. However it is possible to define two distributions $T_{0}$ (the distribution determined by $E_{3}$ ) and the distribution $T_{1}$ (spanned by $E_{1}$ and $E_{2}$ ), which will play a crucial role in the classification. Computing now all components of the Gauss equation, it then follows 
by a long but straightforward computation that the functions $\lambda, a_{1}, \ldots, c_{3}$ have to satisfy the following system of partial differential equations:

$$
\begin{aligned}
& E_{2}\left(a_{1}\right)-E_{1}\left(b_{1}\right)=2 \lambda^{2}+a_{1}^{2}+b_{1}^{2}+b_{2} c_{1}-b_{2} a_{3}+a_{2} b_{3}-a_{3} c_{1}, \\
& E_{3}\left(a_{2}\right)-E_{1}\left(c_{2}\right)=a_{2}^{2}+c_{2}^{2}+b_{2} c_{1}+b_{2} a_{3}-a_{1} c_{3}+a_{3} c_{1}, \\
& E_{2}\left(a_{2}\right)-E_{1}\left(b_{2}\right)=b_{1} a_{3}+b_{1} b_{2}+a_{1} a_{2}-a_{1} b_{3}+c_{2} b_{2}-c_{2} a_{3}, \\
& E_{3}\left(a_{1}\right)-E_{1}\left(c_{1}\right)=a_{2} c_{3}-a_{3} c_{2}+a_{1} a_{2}+b_{1} c_{1}+c_{1} c_{2}+b_{1} a_{3}, \\
& E_{2}\left(c_{2}\right)-E_{3}\left(b_{2}\right)=b_{1} c_{3}-b_{3} c_{1}-a_{2} b_{2}+a_{2} c_{1}-b_{2} b_{3}-c_{2} c_{3}, \\
& E_{2}\left(c_{1}\right)-E_{3}\left(b_{1}\right)=b_{3} c_{2}-c_{3} b_{2}+a_{1} c_{1}-a_{1} b_{2}-c_{1} c_{3}-b_{1} b_{3}, \\
& E_{3}\left(a_{3}\right)-E_{1}\left(c_{3}\right)=a_{1} c_{2}-a_{2} c_{1}+a_{2} a_{3}+a_{3} b_{3}+b_{3} c_{1}+c_{2} c_{3}, \\
& E_{1}\left(b_{3}\right)-E_{2}\left(a_{3}\right)=b_{1} a_{2}-a_{1} b_{2}-a_{1} a_{3}-b_{1} b_{3}+a_{3} c_{3}-c_{3} b_{2}, \\
& E_{3}\left(b_{3}\right)-E_{2}\left(c_{3}\right)=b_{3}^{2}+c_{3}^{2}+a_{3} b_{2}-a_{3} c_{1}+b_{1} c_{2}-b_{2} c_{1} .
\end{aligned}
$$

The number of unknowns in the above equations can be reduced using that $K$ is a Codazzi tensor with respect to the affine metric. In particular, we can show the following:

Lemma 5. We have

(i) $c_{2}=c_{3}=0$,

(ii) $c_{1}=\frac{1}{3} b_{2}$,

(iii) $a_{3}=-b_{2}$,

(iv) $b_{3}=a_{2}$.

Moreover, the function $\lambda$ satisfies the following system of differential equations:

$$
\begin{aligned}
& E_{1}(\lambda)=-3 b_{1} \lambda, \\
& E_{2}(\lambda)=3 a_{1} \lambda, \\
& E_{3}(\lambda)=a_{2} \lambda .
\end{aligned}
$$

Proof. From

$$
\left(\hat{\nabla}_{E_{1}} K\right)\left(E_{3}, E_{3}\right)=\left(\hat{\nabla}_{E_{3}} K\right)\left(E_{1}, E_{3}\right),
$$

it follows immediately that $\hat{\nabla}_{E_{3}} E_{3}=0$. Consequently $c_{2}=c_{3}=0$. As

$$
\left(\hat{\nabla}_{E_{1}} K\right)\left(E_{2}, E_{3}\right)=\left(\hat{\nabla}_{E_{2}} K\right)\left(E_{1}, E_{3}\right),
$$

we deduce that $K\left(E_{2}, \hat{\nabla}_{E_{1}} E_{3}\right)=K\left(E_{1}, \hat{\nabla}_{E_{2}} E_{3}\right)$. As $\lambda \neq 0$, this implies that

$$
\begin{aligned}
& b_{2}=-a_{3}, \\
& b_{3}=a_{2} .
\end{aligned}
$$

The other equations are obtained similarly.

As the connection is torsion free and thus

$$
E_{1}\left(E_{2}(\lambda)\right)-E_{2}\left(E_{1}(\lambda)\right)=\left(\hat{\nabla}_{E_{1}} E_{2}-\hat{\nabla}_{E_{2}} E_{1}\right) \lambda,
$$

we deduce from the differential equations for $\lambda$ that

$$
E_{1}\left(a_{1}\right)+E_{2}\left(b_{1}\right)=-\frac{2}{3} a_{2} b_{2} .
$$

Using the previous lemma, the differential equations given by (49) to (57) now reduce to

$$
\begin{aligned}
& E_{2}\left(a_{1}\right)-E_{1}\left(b_{1}\right)=2 \lambda^{2}+a_{1}^{2}+b_{1}^{2}+\frac{5}{3} b_{2}^{2}+a_{2}^{2}, \\
& E_{3}\left(a_{2}\right)=a_{2}^{2}-b_{2}^{2}, \\
& E_{2}\left(a_{2}\right)-E_{1}\left(b_{2}\right)=0, \\
& E_{3}\left(a_{1}\right)-\frac{1}{3} E_{1}\left(b_{2}\right)=a_{1} a_{2}-\frac{2}{3} b_{1} b_{2}, \\
& E_{3}\left(b_{2}\right)=2 a_{2} b_{2}, \\
& \frac{1}{3} E_{2}\left(b_{2}\right)-E_{3}\left(b_{1}\right)=-\frac{2}{3} a_{1} b_{2}-b_{1} a_{2}, \\
& E_{1}\left(a_{2}\right)+E_{2}\left(b_{2}\right)=0 .
\end{aligned}
$$


Now, we want to solve the above system of differential equations explicitly. In order to do so, we remark that by dividing $M$ into several parts (and ignoring a set of measure zero), we may assume that either

(i) the distribution spanned by $E_{1}$ and $E_{2}$ is integrable on $M$,

(ii) the distribution spanned by $E_{1}$ and $E_{2}$ is nowhere integrable.

We start with the first case. As the distribution is integrable, it follows that $b=0$. In this case, we have the following theorem:

\section{Theorem 7 .}

(i) Let $\phi: M^{2} \rightarrow \mathbb{R}^{3}:(u, v) \mapsto(u, v, f(u, v))$ be an improper affine sphere with affine normal $(0,0,1)$. Then,

$$
F(u, v, t)=\left(u, v, t, f(u, v)+\frac{1}{2} t^{2}\right)
$$

is an improper affine sphere of Type (iv) for which the distribution $T_{1}$ is integrable.

(ii) Let $\phi: M^{2} \rightarrow \mathbb{R}^{3}:(u, v) \mapsto(u, v, f(u, v))$ be a proper elliptic affine sphere. Then,

$$
F(u, v, t)=\left(t \phi(u, v), \frac{1}{2} t^{2}\right),
$$

is an improper affine sphere of Type (iv) for which the distribution $T_{1}$ is integrable.

(iii) Conversely, every 3-dimensional improper afffine hypersphere of Type (iv) for which the distribution $T_{1}$ is integrable can be locally obtained in this way.

Proof. As $b_{2}=0$, we have that both the distributions $T_{0}$ and $T_{1}$ are integrable. Hence there exist coordinates $t, u, v$ such that $E_{3}=\partial_{t}$ and $\partial_{u}$ and $\partial_{v}$ span the distribution $T_{1}$. It follows from (60), (61) and (65) that the function $a_{2}$ is determined by

$$
\begin{aligned}
& \partial_{t} a_{2}=a_{2}^{2}, \\
& \partial_{u} a_{2}=\partial_{\imath} a_{2}=0 .
\end{aligned}
$$

First, we assume that $a_{2}$ vanishes identically. In this case it follows that $F\left(t_{0}, u, v\right)$ defines an improper affine sphere with affine normal $\xi$. Moreover, we have that along this surface, $E_{3}$ is a constant vector. Hence by applying an affine transformation, we may assume that $F\left(t_{0}, u, v\right)=(u, v, f(u, v), 0), \xi=$ $(0,0,1,0)$ and $E_{3}\left(t_{0}, u, v\right)=(0,0,0,1)$. Taking into account that

$$
\frac{\partial^{2}}{\partial t^{2}} F=D_{E_{3}} E_{3}=\xi
$$

it follows that $F$ is congruent with

$$
F(t, u, v)=\left(u, v, t, f(u, v)+\frac{1}{2} t^{2}\right)
$$

This completes the proof in this case.

By the differential equation for $a_{2}$, we may now assume that $a_{2}$ is a nonvanishing function of $t$. If necessary by replacing $E_{3}$ with $-E_{3}$, we may assume that $a_{2}$ is positive. Solving the differential equation explicitly, we may assume that after a translation in the $t$-coordinate we have that $\frac{1}{a_{2}}=-t$. It now follows that $F\left(t_{0}, u, v\right)$ is an elliptic proper affine sphere with affine normal $E_{3}+\frac{1}{a_{2}} \xi$. Using again that

$$
\frac{\partial^{2}}{\partial t^{2}} F=D_{E_{3}} E_{3}=\xi
$$

it now follows easily that we can write

$$
F(t, u, v)=\left(t \phi(u, v), \frac{1}{2} t^{2}\right)
$$

where $(u, v) \mapsto(u, v, \phi(u, v))$ defines an elliptic proper affine sphere in $\mathbb{R}^{3}$.

Next, we focus on the case that the distribution $T_{1}$ is nowhere integrable, implying that the function $b_{2}$ is a nowhere vanishing function. In order to solve the system of differential equations (58)-(65) we 
introduce functions $f, \alpha_{1}, \alpha_{2}, \beta_{1}, \beta_{2}$ on $M$ by

$$
\begin{aligned}
& f=\frac{a_{2}}{a_{2}^{2}+b_{2}^{2}}, \\
& \beta_{1}=E_{1}(f), \\
& \beta_{2}=E_{2}(f), \\
& \left(\alpha_{1}+i \alpha_{2}\right)^{3}=\frac{1}{\lambda\left(a_{2}-i b_{2}\right)^{2}},
\end{aligned}
$$

where $\alpha_{1}$ and $\alpha_{2}$ are well defined up to multiplication by $e^{\frac{2}{3} \pi i}$, due to the nonvanishing of $b_{2}$. We then have that

Consequently, we deduce that

$$
E_{3}(f)=E_{3}\left(\frac{a_{2}}{a_{2}^{2}+b_{2}^{2}}\right)=-1
$$

$$
\begin{aligned}
E_{3}\left(\beta_{1}\right) & =E_{3}\left(E_{1}(f)\right) \\
& =E_{1}\left(E_{3}(f)\right)+\left(\hat{\nabla}_{E_{3}} E_{1}-\hat{\nabla}_{E_{1}} E_{3}\right) f \\
& =-\frac{2}{3} b_{2} \beta_{2}+a_{2} \beta_{1} .
\end{aligned}
$$

Similarly, we deduce that

$$
\begin{aligned}
& E_{3}\left(\beta_{2}\right)=\frac{2}{3} b_{2} \beta_{1}+a_{2} \beta_{2}, \\
& E_{1}\left(\beta_{2}\right)-E_{2}\left(\beta_{1}\right)=2 b_{2}-a_{1} \beta_{1}-b_{1} \beta_{2}, \\
& E_{3}\left(\alpha_{1}\right)=-a_{2} \alpha_{1}-\frac{2}{3} b_{2} \alpha_{2}, \\
& E_{3}\left(\alpha_{2}\right)=\frac{2}{3} b_{2} \alpha_{1}-a_{2} \alpha_{2}, \\
& E_{1}\left(\alpha_{1}\right)-E_{2}\left(\alpha_{2}\right)=a_{1} \alpha_{2}+b_{1} \alpha_{1}, \\
& E_{1}\left(\alpha_{2}\right)+E_{2}\left(\alpha_{1}\right)=-a_{1} \alpha_{1}+b_{1} \alpha_{2} .
\end{aligned}
$$

A straightforward computation, using the above differential equations, then shows that the vector fields $T, U$ and $V$ defined by

$$
\begin{aligned}
& T=E_{3}, \\
& U=\alpha_{1} E_{1}+\alpha_{2} E_{2}+\left(\alpha_{1} \beta_{1}+\alpha_{2} \beta_{2}\right) E_{3}, \\
& V=-\alpha_{2} E_{1}+\alpha_{1} E_{2}+\left(-\alpha_{2} \beta_{1}+\alpha_{1} \beta_{2}\right) E_{3},
\end{aligned}
$$

satisfy that $[T, U]=[T, V]=[U, V]=0$. Consequently, we have that there exist local coordinates $t, u$ and $v$ such that $\frac{\partial}{\partial t}=T=E_{3}, \frac{\partial}{\partial u}=U$ and $\frac{\partial}{\partial v}=V$.

Solving now first (60) and (63), which can be rewritten as

$$
\frac{\partial}{\partial t}\left(a_{2}+i b_{2}\right)=\left(a_{2}+i b_{2}\right)^{2},
$$

we find that there exists functions $C_{1}$ and $C$ depending on the variables $u$ and $v$ such that

$$
\begin{aligned}
& a_{2}=\frac{-t+C_{1}}{\left(-t+C_{1}\right)^{2}+C^{2}}, \\
& b_{2}=-\frac{C}{\left(-t+C_{1}\right)^{2}+C^{2}} .
\end{aligned}
$$

From the definition of $\beta_{1}$ and $\beta_{2}$ it then follows that $C_{1}$ is a constant and thus by a translation of the $t$-coordinate we may assume that $C_{1}$ vanishes identically. Solving now the differential equation for $\lambda$, we find that there exist a function $D$ depending on $u$ and $v$ such that

$$
\lambda=\frac{e^{D}}{\sqrt{t^{2}+C^{2}}} \text {. }
$$

Solving the differential equations (61) and (65) for $\beta_{1}$ and $\beta_{2}$ we deduce that

$$
\begin{array}{r}
\beta_{1}=-\frac{\alpha_{2} \partial_{u} C+\alpha_{1} \partial_{v} C}{\alpha_{1}^{2}+\alpha_{2}^{2}}, \\
\beta_{2}=\frac{\alpha_{1} \partial_{u} C-\alpha_{2} \partial_{v} C}{\alpha_{1}^{2}+\alpha_{2}^{2}} .
\end{array}
$$

Using then the differential equations for $\lambda$ to define the functions $a_{1}$ and $b_{1}$, we find after a straightforward computation that the differential equations (62) and (64) become trivially satisfied. On the other hand, 
the differential equations (58) and (59) imply that the functions $C$ and $D$ satisfy the following system of differential equations:

$$
\begin{aligned}
& \triangle C=-2 C E^{-\frac{2}{3} D}, \\
& \triangle D=\left(3+6 E^{2 D}\right) E^{-\frac{2}{3} D} .
\end{aligned}
$$

We now can formulate the following theorem:

Theorem 8. Let $S$ be a open domain in $\mathbb{R}^{2}$ and let $(C, D)$ be a solution of the system (73)-(74) such that $C$ is a nowhere vanishing function on $S$. Then $S \times \mathbb{R}$ can be immersed as an improper affine hypersphere of Type (iv). Conversely every improper affine hypersphere of Type (iv) for which the distribution $T_{1}$ is nowhere integrable can be characterized by giving a pair of nonzero functions satsifying the above system of elliptic equations.

Proof. We define functions $a_{2}, b_{2}, \alpha_{1}, \alpha_{2}, \lambda, \beta_{1}, \beta_{2}, a_{1}$ and $b_{1}$ as described in the previous equations. We define vector fields $E_{1}, E_{2}$ and $E_{3}$ on $S \times \mathbb{R}$ by

$$
\begin{aligned}
& E_{1}=\frac{\alpha_{1}}{\alpha_{1}^{2}+\alpha_{2}^{2}} \partial_{u}-\frac{\alpha_{2}}{\alpha_{1}^{2}+\alpha_{2}^{2}} \partial_{v}-\beta_{1} \partial_{t} \\
& E_{2}=\frac{\alpha_{2}}{\alpha_{1}^{2}+\alpha_{2}^{2}} \partial_{u}+\frac{\alpha_{1}}{\alpha_{1}^{2}+\alpha_{2}^{2}} \partial_{v}-\beta_{2} \partial_{t} \\
& E_{3}=\partial_{t} .
\end{aligned}
$$

We introduce a metric $h$ on $S \times I$ by the assumption that $E_{1}, E_{2}$ and $E_{3}$ form an orthonormal basis and a difference tensor $K$ by

$$
\begin{array}{lll}
K\left(E_{1}, E_{1}\right)=\lambda E_{1}, & K\left(E_{1}, E_{2}\right)=-\lambda E_{2}, & K\left(E_{3}, E_{1}\right)=0, \\
K\left(E_{2}, E_{2}\right)=-\lambda E_{1}+\mu E_{2}, & K\left(E_{2}, E_{3}\right)=0, & K\left(E_{3}, E_{3}\right)=0 .
\end{array}
$$

We also define a shape operator $S=0$. It is then straightforward to check that $h, K$ and $S$ satisfy all the conditions of the fundamental theorem of [5]. Consequently $S \times I$ can be immersed as an improper affine hypersphere with affine metric $h$ and difference tensor $K$. Clearly, it is an improper affine hypersphere of Type (iv). It is straightforward to check that the distribution $T_{1}$ is nowhere integrable. The converse statement was proved immediately before we stated the theorem.

\section{Affine hyperspheres of Type (v)}

Finally, in this section, we assume that $M$ is an affine hypersphere of Type (vi). It is easy to see that this implies that at each point $p$ there exists an orthonormal basis $\left\{e_{1}, e_{2}, e_{3}\right\}$ such that

$$
\begin{array}{lll}
K\left(e_{1}, e_{1}\right)=\lambda_{1} e_{1}, & K\left(e_{1}, e_{2}\right)=\lambda_{2} e_{2}, & K\left(e_{3}, e_{1}\right)=\lambda_{3} e_{3}, \\
K\left(e_{2}, e_{2}\right)=\lambda_{2} e_{1}, & K\left(e_{2}, e_{3}\right)=0, & K\left(e_{3}, e_{3}\right)=\lambda_{3} e_{3},
\end{array}
$$

where $0<\lambda_{1}=-\lambda_{2}-\lambda_{3}$ and $0 \neq \lambda_{2} \neq \lambda_{3} \neq 0$.

By a straightforward computation we obtain the following:

Lemma 6. Let $\left\{e_{1}, e_{2}, e_{3}\right\}$ be the orthonormal basis defined previously. Then it follows that

$$
\begin{array}{lll}
\widehat{\operatorname{Ric}}\left(e_{1}, e_{1}\right)=\epsilon+\lambda_{2}^{2}+\lambda_{3}^{2}+\lambda_{2} \lambda_{3}, & \widehat{\operatorname{Ric}}\left(e_{1}, e_{2}\right)=0, & \widehat{\operatorname{Ric}}\left(e_{3}, e_{1}\right)=0, \\
\widehat{\operatorname{Ric}}\left(e_{2}, e_{2}\right)=\epsilon+\lambda_{2}^{2}, & \widehat{\operatorname{Ric}}\left(e_{2}, e_{3}\right)=0, & \widehat{\operatorname{Ric}}\left(e_{3}, e_{3}\right)=\epsilon+\lambda_{3}^{2} .
\end{array}
$$

Using the various conditions on $\lambda_{2}$ and $\lambda_{3}$, we see that the 1-1 symmetric tensor field $P$ associated with the Ricci tensor has at each point three different eigenvalues, all with multiplicity 1 . Hence there exist orthonormal vector fields $\left\{E_{1}, E_{2}, E_{3}\right\}$ defined on a neighborhood of the point $p$ and a non vanishing differentiable functions $\lambda_{2}, \lambda_{3}$, with $\lambda_{2}+\lambda_{3}<0$ and $\lambda_{2}-\lambda_{3} \neq 0$ such that

$$
\begin{array}{lll}
K\left(E_{1}, E_{1}\right)=-\left(\lambda_{2}+\lambda_{3}\right) E_{1}, & K\left(E_{1}, E_{2}\right)=\lambda_{2} E_{2}, & K\left(E_{3}, E_{1}\right)=\lambda_{3} E_{3}, \\
K\left(E_{2}, E_{2}\right)=\lambda_{2} E_{1}, & K\left(E_{2}, E_{3}\right)=0, & K\left(E_{3}, E_{3}\right)=\lambda_{3} E_{1} .
\end{array}
$$


Computing now all components of the Gauss equation, it then follows by a long but straightforward computation that the functions $\lambda_{2}, \lambda_{3}, a_{1}, \ldots, c_{3}$ have to satisfy the following system of partial differential equations:

$$
\begin{aligned}
& E_{2}\left(a_{1}\right)-E_{1}\left(b_{1}\right)=\epsilon+2 \lambda_{2}+\lambda_{2} \lambda_{3}+a_{1}^{2}+b_{1}^{2}+b_{2} c_{1}-b_{2} a_{3}+a_{2} b_{3}-a_{3} c_{1}, \\
& E_{3}\left(a_{2}\right)-E_{1}\left(c_{2}\right)=\epsilon+2 \lambda_{3}^{2}+\lambda_{2} \lambda_{3}+a_{2}^{2}+c_{2}^{2}+b_{2} c_{1}+b_{2} a_{3}-a_{1} c_{3}+a_{3} c_{1}, \\
& E_{2}\left(a_{2}\right)-E_{1}\left(b_{2}\right)=b_{1} a_{3}+b_{1} b_{2}+a_{1} a_{2}-a_{1} b_{3}+c_{2} b_{2}-c_{2} a_{3}, \\
& E_{3}\left(a_{1}\right)-E_{1}\left(c_{1}\right)=a_{2} c_{3}-a_{3} c_{2}+a_{1} a_{2}+b_{1} c_{1}+c_{1} c_{2}+b_{1} a_{3}, \\
& E_{2}\left(c_{2}\right)-E_{3}\left(b_{2}\right)=b_{1} c_{3}-b_{3} c_{1}-a_{2} b_{2}+a_{2} c_{1}-b_{2} b_{3}-c_{2} c_{3}, \\
& E_{2}\left(c_{1}\right)-E_{3}\left(b_{1}\right)=b_{3} c_{2}-c_{3} b_{2}+a_{1} c_{1}-a_{1} b_{2}-c_{1} c_{3}-b_{1} b_{3}, \\
& E_{3}\left(a_{3}\right)-E_{1}\left(c_{3}\right)=a_{1} c_{2}-a_{2} c_{1}+a_{2} a_{3}+a_{3} b_{3}+b_{3} c_{1}+c_{2} c_{3}, \\
& E_{1}\left(b_{3}\right)-E_{2}\left(a_{3}\right)=b_{1} a_{2}-a_{1} b_{2}-a_{1} a_{3}-b_{1} b_{3}+a_{3} c_{3}-c_{3} b_{2}, \\
& E_{3}\left(b_{3}\right)-E_{2}\left(c_{3}\right)=\epsilon-\lambda_{2} \lambda_{3}+b_{3}^{2}+c_{3}^{2}+a_{3} b_{2}-a_{3} c_{1}+b_{1} c_{2}-b_{2} c_{1} .
\end{aligned}
$$

The number of unknowns in the above equations can be reduced using that $K$ is a Codazzi tensor with respect to the affine metric. In particular, by a straightforward computation we obtain the following:

Lemma 7. There exists a local function $c$ such that

(i) $b_{2}=c_{1}=a_{3}=0$,

(ii) $a_{2}=\left(1-\frac{\lambda_{3}}{\lambda_{2}}\right) b_{3}$,

(iii) $a_{1}=\left(\frac{\lambda_{2}}{\lambda_{3}}-1\right) c_{3}$,

(iv) $c_{2}=c \lambda_{3}$

(v) $b_{1}=c \lambda_{2}$.

Moreover, the functions $\lambda_{2}$ and $\lambda_{3}$ satisfy the following system of differential equations:

$$
\begin{aligned}
& E_{1}\left(\lambda_{2}\right)=-c \lambda_{2}\left(3 \lambda_{2}+\lambda_{3}\right), \\
& E_{2}\left(\lambda_{2}\right)=3 c_{3} \lambda_{2}\left(\frac{\lambda_{2}}{\lambda_{3}}-1\right), \\
& E_{3}\left(\lambda_{2}\right)=b_{3}\left(\lambda_{2}-\lambda_{3}\right), \\
& E_{1}\left(\lambda_{3}\right)=-c \lambda_{3}\left(3 \lambda_{3}+\lambda_{2}\right), \\
& E_{2}\left(\lambda_{3}\right)=c_{3}\left(\lambda_{2}-\lambda_{3}\right), \\
& E_{3}\left(\lambda_{3}\right)=3 b_{3} \lambda_{3}\left(1-\frac{\lambda_{3}}{\lambda_{2}}\right) .
\end{aligned}
$$

Using the previous lemma, the differential equations given by (77) to (83) now imply that

$$
\begin{array}{r}
E_{2}(c)=0, \\
E_{3}(c)=0, \\
E_{1}\left(c_{3}\right)=-c c_{3} \lambda_{2}, \\
E_{1}\left(b_{3}\right)=-b_{3} c \lambda_{3}, \\
E_{3}\left(c_{3}\right)=3 b_{3} c_{3}, \\
E_{2}\left(b_{3}\right)=-3 b 3 c_{3} .
\end{array}
$$

If we now introduce a function $\alpha$ by

$$
E_{1}(c)=\alpha,
$$

we deduce from (75) and (76) that

$$
\begin{aligned}
E_{2}\left(c_{3}\right)= & \frac{1}{\lambda_{2} \lambda_{3}\left(\lambda_{2}-\lambda_{3}\right)}\left(c_{3}^{2}\left(-\lambda_{2}^{3}+\lambda_{2} \lambda_{3}^{2}\right)+\lambda_{3}^{3}\left(b_{3}^{2}\left(\lambda_{2}-\lambda_{3}\right)\right.\right. \\
& \left.+\lambda_{2}\left(\epsilon+\lambda_{2}\left(\alpha-\left(c^{2}-1\right)\left(2 \lambda_{2}+\lambda_{3}\right)\right)\right)\right) \\
E_{3}\left(b_{3}\right)= & \frac{1}{\lambda_{2} \lambda_{3}\left(\lambda_{2}-\lambda_{3}\right)}\left(c_{3}^{2} \lambda_{2}^{2}\left(-\lambda_{2}+\lambda_{3}\right)+\lambda_{3}\left(b_{3}^{2}\left(\lambda_{2}^{2}-\lambda_{3}^{2}\right)\right.\right. \\
& \left.+\lambda_{2}^{2}\left(\epsilon+\lambda_{3}\left(\alpha-\left(c^{2}-1\right)\left(\lambda_{2}+2 \lambda_{3}\right)\right)\right)\right)
\end{aligned}
$$


We now compute some integrability conditions. As $\hat{\nabla}$ is torsion free, we know that for any function $f$, the following equations are satisfied:

$$
\begin{aligned}
& 0=E_{1}\left(E_{2}(f)\right)-E_{2}\left(E_{1}(f)\right)-\left(\hat{\nabla}_{E_{1}} E_{2}\right)(f)+\left(\hat{\nabla}_{E_{2}} E_{1}\right)(f), \\
& 0=E_{1}\left(E_{3}(f)\right)-E_{3}\left(E_{1}(f)\right)-\left(\hat{\nabla}_{E_{1}} E_{3}\right)(f)+\left(\hat{\nabla}_{E_{3}} E_{1}\right)(f), \\
& 0=E_{2}\left(E_{3}(f)\right)-E_{3}\left(E_{2}(f)\right)-\left(\hat{\nabla}_{E_{2}} E_{3}\right)(f)+\left(\hat{\nabla}_{E_{3}} E_{2}\right)(f) .
\end{aligned}
$$

It is straightforward to check that applying the above principle for the functions $\lambda_{2}$ and $\lambda_{3}$ does not yield any new equations. However, applying this principle for the functions $c, c_{3}$ and $b_{3}$ yields the following system of differential equations for the function $\alpha$ :

$$
\begin{aligned}
E_{2}(\alpha)= & -\alpha c_{3}\left(1-\frac{\lambda_{2}}{\lambda_{3}}\right), \\
E_{3}(\alpha)= & \alpha b_{3}\left(1-\frac{\lambda_{3}}{\lambda_{2}}\right) \\
E_{1}(\alpha)= & -\frac{1}{\lambda_{2}^{2} \lambda_{3}^{2}}\left(c \left(4 c_{3}^{2} \lambda_{2}^{2}\left(\lambda_{2}-\lambda_{3}\right)^{2}+\lambda_{3}^{2}\left(4 b_{3}^{2}\left(\lambda_{2}-\lambda_{3}\right)^{2}\right.\right.\right. \\
& \left.\left.\left.+\lambda_{2}^{2}\left(4 \epsilon-3 \alpha\left(\lambda_{2}+\lambda_{3}\right)+4\left(c^{2}-1\right)\left(\lambda_{2}^{2}+\lambda_{2} \lambda_{3}+\lambda_{3}^{2}\right)\right)\right)\right)\right),
\end{aligned}
$$

of which one can check that the integrability conditions are satisfied.

We now consider several cases. By restricting to an open dense subset of $M$, we may assume that one of the following holds:

(i) the functions $b_{3}$ and $c_{3}$ are non vanishing functions. In this case we introduce positive, nonvanishing functions $\rho_{1}, \rho_{2}$ and $\rho_{3}$ by

$$
\begin{aligned}
& \rho_{1}=\left(\lambda_{2} \lambda_{3}\right)^{-\frac{1}{4}}, \\
& \rho_{2}=c_{3}^{-\frac{1}{3}} \lambda_{2}^{-\frac{1}{4}} \lambda_{3}^{\frac{1}{12}}, \\
& \rho_{3}=b_{3}^{-\frac{1}{3}} \lambda_{3}^{-\frac{1}{4}} \lambda_{2}^{\frac{1}{12}} .
\end{aligned}
$$

(ii) the function $c_{3}$ vanishes identically on $M$ and the function $b_{3}$ is a non vanishing function. In this case we introduce positive, nonvanishing functions $\rho_{1}, \rho_{2}$ and $\rho_{3}$ by

$$
\begin{aligned}
& \rho_{1}=\left(\lambda_{2} \lambda_{3}\right)^{-\frac{1}{4}}, \\
& \rho_{3}=b_{3}^{-\frac{1}{3}} \lambda_{3}^{-\frac{1}{4}} \lambda_{2}^{\frac{1}{12}},
\end{aligned}
$$

and the function $\rho_{2}$ is determined by the following integrable system

$$
\begin{aligned}
& E_{1}\left(\rho_{2}\right)=c \lambda_{2} \rho_{2}, \\
& E_{2}\left(\rho_{2}\right)=0, \\
& E_{3}\left(\rho_{2}\right)=-b_{3} .
\end{aligned}
$$

(iii) both the functions $b_{3}$ and $c_{3}$ vanish identically in which case we introduce the function $\rho_{1}$ by

$$
\rho_{1}=\left(\lambda_{2} \lambda_{3}\right)^{-\frac{1}{4}}
$$

and the functions $\rho_{2}$ and $\rho_{3}$ are determined by the following integrable system of differential equations:

$$
\begin{array}{ll}
E_{1}\left(\rho_{1}\right)=-c \lambda_{2} \rho_{2}, & E_{1}\left(\rho_{3}\right)=-c \lambda_{3} \rho_{3}, \\
E_{2}\left(\rho_{2}\right)=0, & E_{2}\left(\rho_{3}\right)=0, \\
E_{3}\left(\rho_{3}\right)=0, & E_{3}\left(r h o_{3}\right)=0 .
\end{array}
$$

In all three cases, it follows that

$$
\left[\rho_{1} E_{1}, \rho_{2} E_{2}\right]=\left[\rho_{2} E_{2}, \rho_{3} E_{3}\right]=\left[\rho_{1} E_{1}, \rho_{3} E_{3}\right]=0,
$$


which implies that there exist local coordinates $u, v$ and $w$ such that

$$
\begin{aligned}
& \frac{\partial}{\partial u}=\rho_{1} E_{1}, \\
& \frac{\partial}{\partial v}=\rho_{2} E_{2}, \\
& \frac{\partial}{\partial w}=\rho_{3} E_{3} .
\end{aligned}
$$

Replacing now the $E_{1}, 2$ and $E_{3}$ derivatives by derivatives with respect to $u, v$ and $w$, we obtain a completely integrable system. Using then the existence and uniqueness theorem of affine immersions, we immediately obtain the following:

Theorem 9. Let $M$ be an affine hypersphere of Type (v). Then, $M$ is completely determined by giving initial conditions for the functions $\lambda_{2}, \lambda_{3}, b_{3}, c_{3}, c$ and $\alpha$ at a given point. Conversely,

(i) Given initial values for $\lambda_{2}$ and $\lambda_{3}$ at a point, we can construct an affine hypersphere of Type (v)

by assuming that $b_{3}=c_{3}=0, \alpha=\left(c^{2}-1\right) 2\left(\lambda_{2}+\lambda_{3}\right)$ and $\epsilon+\lambda_{3} \lambda_{2}\left(c^{2}-1\right)=0$

(ii) Given initial values for $\lambda_{2}, \lambda_{3}, b_{3} \neq 0, c_{3} \neq 0, c$ and $\alpha$ at a point, we can construct an affine hypersphere of Type (v)

(iii) Given initial values for $b_{3}, \lambda_{2}, \lambda_{3}$ and $c$ at a point, we can construct an affine hypersphere of Type (v) by assuming that $c_{3}=0$ and $\alpha$ is determined by

$$
b_{3}^{2}\left(\lambda_{2}-\lambda_{3}\right)+\lambda_{2}\left(\epsilon+\lambda_{2}\left(\alpha-\left(c^{2}-1\right)\left(2 \lambda_{2}+\lambda_{3}\right)\right)\right) .
$$

\section{REFERENCES}

[1] R. L. Bryant Second order families of special Lagrangian 3-folds, preprint

[2] E. Calabi. Complete affine hyperspheres. I. Sympos. math., 10(1972):19-38.

[3] B.-Y. Chen. Some pinching and classification theorems for minimal submanifolds. Arch. Math., 60(1993):568-578.

[4] S. Y. Cheng and S. T. Yau. Complete affine hypersurfaces. I: The completeness of affine metrics. Commun. Pure Appl. Math., 39(1986): 839-866.

[5] F. Dillen, K. Nomizu and L. Vrancken. Conjugate connections and radon's theorem in affine differential geometry. Monatsh. Math., 109(1990): 221-235.

[6] F. Dillen and L. Vrancken. Calabi type composition of affine spheres. Differential Geometry and its applications, 4(1994): 303-328.

[7] M. Kriele, C. Scharlach and L. Vrancken. An extremal class of 3-dimensional elliptic affine spheres. Hokkaido Math. $J ., 30(2001): 1-23$.

[8] M. Kriele and L. Vrancken. Lorentzian affine hyperspheres with constant sectional curvature. Trans. Amer. Math. Soc., 352(2000): 1581-1599.

[9] M. Kriele and L. Vrancken An extremal class of 3-dimensional hyperbolic affine spheres. Geom. Dedicata, 77(1999): $239-252$.

[10] S. Hiepko, Eine innere Kennzeichung der verzerrten Produkte. Math. Ann., 241(1979): 209-215.

[11] A. M. Li. Calabi conjecture on hyperbolic affine hyperspheres. II. Math. Ann., 293(1992): 485-493.

[12] A. M. Li, U. Simon and L. Vrancken Affine spheres with constant affine sectional curvature. Math. Z., 206(1991): $651-658$.

[13] A. M. Li, U. Simon and G. Zhao. Global Affine Differential Geometry of Hypersurfaces, volume 11 of De Gruyter Expositions in Mathematics. Walter De Gruyter, Berlin-New York, 1993.

[14] K. Nomizu and T. Sasaki. Affine Differential Geometry. Cambridge University press, Cambridge, 1994.

[15] A.V. Pogorelov. On the improper convex affine hyperspheres. Geometriae dedicata, 1(1972):33-46.

[16] C. Rodriguez Montealegre and L. Vrancken. Lagrangian submanifolds of the three dimensional complex projective space J. Math. Soc. Japan, 53(2001), to appear.

[17] T. Sasaki. Hyperbolic affine hyperspheres. Nagoya Math. J., 77(1980): 107-123.

[18] C. Scharlach, U. Simon, L. Verstraelen, and L. Vrancken. A new intrinsic curvature invariant for centroaffine hypersurfaces. Beiträge zur Algebra und Geometrie, 38(1997): 437-458.

[19] L. Vrancken. The Magid-Ryan conjecture for equiaffine hyperspheres with constant sectional curvature, Journal of Differential Geometry, to appear. 